\title{
Transtorno do Espectro Autista: uma abordagem sobre as políticas públicas e 0 acesso à sociedade
}

\author{
Autism Spectrum Disorder: an approach to public policies and the \\ access to society
}

Recebido em: 02/01/2021 Aceito em: 18/02/2021

\author{
Maria Aparecida NICOLETTI; Fernanda Ramaglia HONDA \\ Departamento de Farmácia, Faculdade de Ciências Farmacêuticas, \\ Universidade de São Paulo. Av. Professor Lineu Prestes, 580, \\ CEP 05508-900. São Paulo, SP, Brasil. \\ E-mail:fernanda.ramaglia.honda@usp.br
}

\section{ABSTRACT}

According to the World Health Organization (WHO), Autistic Spectrum Disorder (ASD) is a set of conditions characterized by some degree of difficulty in social interaction, verbal and non-verbal communication, and specific interests due to some activities performed repeatedly. These characteristics appear in childhood, tend to continue in adolescence, and remain when the individual becomes an adult. ASD generates several difficulties for the patient. Therefore, it is necessary to look at what Brazilian societies, among others, are doing to facilitate the routine of these people. The objective of this work was to search for national and international public policies related to ASD. Thus, national and international laws were reviewed, and articles were consulted in databases, such as SciELO and PubMed. There are policies and the provision of information to citizens, about ASD, in all the Countries surveyed. Still, although the pharmacist is not a professional directly related to the treatment of these patients, the first contact of the family with a child with ASD can be done in pharmacies and/or Health Centers (UBS) spread across Brazil, as the contact with the doctor takes longer, due to the complexity of the Unified Health System (SUS). Thus, the pharmacist needs to be prepared to advise the family and identify signs of ASD in the child. It is also the pharmacist's responsibility to join a multi-professional group to develop a therapeutic plan for the patient, assess all risks, and minimize their symptoms.

Keywords: Autism Spectrum Disorder; public policies; Unified Health System.

\section{RESUMO}

Segundo a Organização Mundial da Saúde (OMS), o Transtorno do Espectro Autista (TEA) é um conjunto de condições caracterizadas por algum grau de dificuldade no convívio social, na comunicação verbal e não verbal e interesses específicos por algumas atividades realizadas de forma repetitiva. Essas características surgem ainda na infância, tendem a continuar na adolescência e permanecem quando o indivíduo se torna adulto. Devido à sua capacidade de gerar dificuldades ao indivíduo, é preciso avaliar o que a sociedade brasileira, entre outras, está fazendo para facilitar a rotina dessas pessoas. Portanto, o objetivo 
deste trabalho foi buscar por políticas públicas nacionais e internacionais relacionados ao TEA. Assim, foi realizada a revisão de leis nacionais e internacionais e consulta de artigos nas bases de dados, como SciELO, PubMed, Web of Science e Science Direct. Em todos os países pesquisados, há o que se evoluir em relação as políticas, bem como a disponibilização de informações aos cidadãos. Ainda, embora o farmacêutico não seja um profissional diretamente relacionado ao tratamento desses pacientes, o primeiro contato da família com uma criança com TEA pode-se dar nas farmácias e/ou Unidades Básicas de Saúde (UBS) espalhadas pelo Brasil, sendo o contato com o médico de acesso mais demorado, devido à complexidade do Sistema Único de Saúde (SUS). Assim, o farmacêutico precisa estar preparado para aconselhar a família e identificar sinais do TEA na criança. Também é de responsabilidade do farmacêutico integrar um grupo multiprofissional, a fim de elaborar um plano terapêutico para o paciente, avaliar todos os riscos e minimizar seus sintomas.

Palavras-chave: Transtorno do Espectro Autista; Políticas Públicas; Sistema Único de Saúde.

\section{INTRODUÇÃO}

Segundo a Organização Mundial da Saúde (OMS), o Transtorno do Espectro Autista (TEA) é um conjunto de condições caracterizadas por algum grau de dificuldade no convívio social, na comunicação verbal e não verbal e interesses específicos por algumas atividades realizadas de forma repetitiva. Essas características surgem ainda na infância e tendem a continuar na adolescência e, ainda, quando o indivíduo se torna adulto. Algumas condições como epilepsia, depressão, ansiedade, e transtorno de déficit de atenção e hiperatividade também podem estar presentes nos autistas (1).

Anteriormente à criação do Sistema Único de Saúde (SUS), famílias de indivíduos com TEA já tinham a necessidade de consultar outras famílias para debater sobre o comportamento dos parentes e sobre quais medidas adotar. Como o Estado brasileiro não dispunha de qualquer tipo de assistência, a solução foi criar associações por iniciativas próprias, de pais e médicos. Então, em 1983, foi criada a Associação dos Amigos dos Autistas de São Paulo, a AMA-SP (2).

Posteriormente à criação do SUS, em 2002, foram criados os Centros de Atenção Psicossocial Infanto-juvenil (CAPSi), que têm como função prover cuidados às crianças e adolescentes portadores de autismo, psicoses e todos que estejam impossibilitados de manter ou estabelecer laços sociais. É um serviço de atenção diária, cujas atividades ofe- recidas são: atendimento individual, grupal e/ou familiar, visitas domiciliares, atividades de inserção social, oficinas terapêuticas, atividades socioculturais e esportivas, atividades externas (3).

Ainda posteriormente à criação dos CAPS, o indivíduo com TEA não era considerado um deficiente perante a legislação, ou seja, não estava assegurado por outras leis, como a Lei $n^{\circ} 8.069 / 1990$, que diz que crianças e adolescentes com deficiências devem receber atendimento diferenciado (4).

Com o passar dos anos, outras instituições semelhantes à AMA-SP foram criadas e se espalharam para o restante do Brasil. Por meio de familiares participantes destas instituições, em 2012, foi sancionada a Lei $n^{\circ} 12.764$, que "Institui a Política Nacional de Proteção dos Direitos da Pessoa com Transtorno do Espectro Autista" (5).

Para muitos, essa lei é vista como um marco histórico, uma vez que além de reconhecer o autista como uma "pessoa com deficiência, para todos os efeitos legais" (Lei $n^{\circ} 12.764, \S 20$ ), também produz incidências em diversos campos, como na esfera assistencial, político/gestora, científico/acadêmica, educacional/pedagógica, bem como no campo dos direitos básicos (6).

Com a publicação da Portaria do Ministério da Saúde $n^{\circ} 324$, de 31 de março de 2016, que aprovou o Protocolo Clínico e Diretrizes Terapêuticas do Comportamento Agressivo como Transtorno do Espectro do Autismo, o diagnóstico passou a ser realizado de forma clínica e diferencial. A diretriz 
complementa que, por se tratar de uma condição que envolve ampla variedade de expressões sintomáticas, é preciso que mais informações sejam fornecidas para a conclusão do diagnóstico. São elas: nível de comunicação verbal e não verbal, nível intelectual, a extensão do campo de interesses, o contexto familiar e educacional, e a capacidade para uma vida autônoma (7).

A diretriz também cita a Linha de Cuidado para a Atenção às Pessoas com Transtornos do Espectro do Autismo ao abordar os tipos de tratamento (8). A primeira opção seria o tratamento não medicamentoso, englobando as seguintes técnicas: (1) Tratamento clínico de base psicanalítica; (2) Análise do Comportamento Aplicada (Applied Behavior Analysis, ABA); (3) Comunicação Suplementar e Alternativa; (4) Integração Sensorial; e (5) Tratamento e Educação para Crianças com Transtornos do Espectro do Autismo (TEACCH, na sigla em inglês) (9).

A Lei brasileira mais recente foi sancionada em janeiro de 2020, também representando um avanço na inclusão do indivíduo com TEA na sociedade. A Lei $n^{\circ} 13.997$ criou a Carteira de Identificação da Pessoa com Transtorno do Espectro Autista (Ciptea), a qual "garante atenção integral, pronto atendimento e prioridade no atendimento e no acesso aos serviços públicos e privados, em especial nas áreas de saúde, educação e assistência social” (10).

Apesar da criação de uma diretriz nacional para o TEA e da publicação do Decreto no 6.949/2009, o qual garante que os indivíduos com TEA tenham o mesmo direito que outras pessoas de exercer seus direitos trabalhistas e sindicais, além de pleno acesso aos serviços de saúde, educação e serviços sociais, sabe-se que muitos ainda encontram dificuldade para matricular-se em escolas, sejam elas públicas ou privadas, o que leva a uma baixa inclusão social e difícil imersão no mercado de trabalho, independentemente do nível socioeconômico (11).

Enquanto isso, na Inglaterra, em 2009, foi aprovado o Autism Act. Esse foi o único ato aprovado específico para uma deficiência e ele coloca duas questões chave: (1) O governo é responsável por produzir e realizar revisões periódicas nas estratégias de auxílio aos indivíduos com TEA; (2) Deve produzir guias para as autoridades locais so- bre como implementar essas estratégias em suas regiões. A primeira versão foi publicada em 2010, passando por uma revisão em 2014 e contando agora com uma versão publicada em 2015 (12).

O Ato de 2009 teve grande importância para a comunidade inglesa, uma vez que muitas áreas do país não contavam com serviços de diagnóstico para adultos (12). Além disso, impôs que o governo criasse e auxiliasse autoridades locais em relação a muitos aspectos envolvendo indivíduos com TEA, como, por exemplo, questões relacionadas ao mercado de trabalho e sistema criminal $(12,13)$.

Outro exemplo é o caso dos Estados Unidos da América (EUA) cuja legislação baseia-se no ato Autism Collaboration, Accountability, Research Education, and Support (CARES) Act, de 2019. Este é um ato que estabelece que o governo seja responsável por oferecer suporte a pesquisas, serviços e outras atividades. A nova versão do ato também amplia as atividades do governo para toda a vida útil do indivíduo com TEA e exige relatórios sobre o bem-estar dessas pessoas $(14,15)$.

O TEA é um assunto que vem tornando-se cada vez mais divulgado na mídia e a legislação brasileira atual não vem acompanhando essa evolução (16). É importante que o Brasil acompanhe outros países e não se torne obsoleto em relação à inclusão destes indivíduos na sociedade. Dessa forma, conhecer o cenário atual e as políticas públicas disponíveis para os indivíduos com TEA e de suas famílias é necessário, para que a sociedade tenha contato com o assunto e se torne participativa no desenvolvimento de ferramentas para a implementação de medidas que facilitem o cuidado e a inclusão de pessoas com TEA.

\section{MÉTODO}

Foi realizada uma revisão bibliográfica do tipo narrativa sobre o tema proposto, utilizando as seguintes bases de dados: Scientific Electronic Library Online (SciELO), US National Library of Medicine - National Institutes of Health (PubMed), Web of Science e Science Direct, por meio das palavras-chave Transtorno do Espectro Autista, políticas públicas e Sistema Único de Saúde (esse último quando aplicável). Além disso, foram con- 
sultados sites de instituições públicas e privadas, nacionais e internacionais, para a busca de legislação e informações relacionadas ao tema.

Os critérios de inclusão consistiram em artigos e/ou estudos e/ou documentos publicados nos últimos 10 anos, nas línguas portuguesa (Brasil e Portugal) e inglesa.

Foram desconsiderados aqueles cuja data de publicação não tenham atendido ao período estabelecido e/ou aos idiomas propostos, exceto se as publicações anteriores ao período estabelecido contribuíssem para o entendimento do cenário de maneira mais abrangente.

Uma vez cumprido os critérios de inclusão mencionados anteriormente, as publicações foram inicialmente analisadas por meio da leitura do seu respectivo título e resumo. Caso seu conteúdo fosse pertinente ao trabalho e trouxesse boas contribuições para a discussão do tema, ele foi então lido na íntegra.

\section{RESULTADOS E DISCUSSÃO}

Políticas Públicas Brasileiras. No Brasil, as políticas públicas para indivíduos com TEA foram implementadas tardiamente. Sem a presença do SUS, ou seja, até 1988, não havia qualquer tipo de atenção a estes indivíduos. Posteriormente à criação do SUS, em 2002, foi assinada a Portaria $n^{\circ}$ 1.635 pelo Ministro da Saúde, a qual incluiu, no Sistema de Informações Ambulatoriais do Sistema Único de Saúde - SIASUS, procedimentos relacionados à pacientes com deficiência mental $\mathrm{e}$ autismo:

"Consiste no conjunto de atividades individuais de estimulação sensorial $e$ psicomotora, realizada por equipe multiprofissional, visando à reeducação das funções cognitivas e sensoriais. Inclui avaliação, estimulação e orientação relacionadas ao desenvolvimento da pessoa portadora de deficiência mental ou com autismo (máximo 20 procedimentos/paciente/mês)" (17).

Entretanto, uma Portaria possui caráter administrativo, não possui a força de uma Lei. Assim, apenas em 2012, ou seja, 24 anos após a criação do SUS, por meio da Lei no 12.764 , que "Institui a Política Nacional de Proteção dos Direitos da Pessoa com Transtorno do Espectro Autista”, surge, então, a primeira política pública para um indivíduo com TEA (5).

Essa Lei foi um marco importante para os direitos dos autistas. Além de considerar que indivíduos com TEA são pessoas com deficiência, para todos os efeitos legais, podendo incluí-los no Decreto $\mathrm{n}^{\circ} 6.949 / 2009$, a mesma também garante que os mesmos devem ter direito à saúde, educação e acesso ao mercado de trabalho. Também prevê punição em caso de a escola recusar a matrícula do aluno autista $(5,11)$.

Ainda, apenas dois anos após a sanção da Lei, o Decreto $\mathrm{n}^{\mathrm{o}} 8.368$, de 2014, entrou em vigor, a fim de regulamentar a Lei ${ }^{\circ} 12.764 / 2012$. A Lei ainda prevê que o indivíduo com TEA, caso comprovada a necessidade de apoio às atividades da rotina, tem direito a um acompanhante e que a instituição de ensino tem o dever de disponibilizar esse acompanhante. Entretanto, nem a Lei nem o Decreto determinam qual deve ser a especialização desse acompanhante (18).

Depois de dois anos após a implementação da Lei foram criadas as Diretrizes de Atenção à Reabilitação da Pessoa com Transtornos do Espectro Autista (19). Ainda, no ano seguinte, foi criada a Linha de Cuidado para a Atenção às Pessoas com Transtornos do Espectro do Autismo e suas Famílias na Rede de Atenção Psicossocial do Sistema Único de Saúde (8).

No quesito defesa de direitos, a Diretriz acima mencionada cita a legislação sobre deficiência: o Decreto $\mathrm{n}^{\circ}$ 6.949, de 2009, que "promulga a Convenção Internacional sobre os Direitos das Pessoas com Deficiência e seu Protocolo Facultativo, assinados em Nova York, em 30 de março de 2007". A Linha de Cuidados cita duas legislações: Lei 10.216, de 2001, que "dispõe sobre o direito das pessoas portadoras de transtornos mentais e redireciona o modelo assistencial em saúde mental", e a Lei 10.708, de 2003, que "institui o auxílio-reabilitação psicossocial para pacientes acometidos de transtornos mentais egressos de internações" $(8,11,19-21)$. 
As publicações convergem em relação a: 1) determinados princípios básicos envolvidos na terapêutica do autismo (tais como a promoção de autonomia, do aprimoramento do desempenho em atividades sociais e cotidianas, a inserção no mercado de trabalho e a integração no cenário comunitário); 2) valorização do papel dos familiares durante todo o programa de tratamento; 3) significativa importância do trabalho colaborativo e Inter setorial; 4) defesa de direitos; e 5) detecção precoce e diagnóstico diferencial (6).

Apesar das divergências (mais relacionadas ao modo de escrita e sua abrangência) e convergências entre os documentos, ambos concordam em um princípio básico fundamental: prover autonomia aos pacientes e proporcionar a reinserção social. Além disso, as discordâncias não atingem pontos cruciais, uma vez que ambos não demonstram concorrência entre os mesmos, mas, sim, complementariedade (6).

Posteriormente aos dois documentos, em 2016, foi aprovado o Protocolo Clínico e Diretrizes Terapêuticas do Comportamento Agressivo como Transtorno do Espectro do Autismo (7). Este é mais um documento apresentado pelo Ministério da Saúde que descreve classificação, diagnóstico, tratamentos e outros tópicos sobre o TEA. Como o próprio documento cita, este é um complemento a outros documentos elaborados pelo Ministério da Saúde:

“[...] Este Protocolo é um complemento às diretrizes clínicas do Ministério da Saúde sobre os Transtornos do Espectro do Autismo (TEA), sobretudo quanto ao uso de condutas terapêuticas em âmbito ambulatorial no que diz respeito à melhoria de condutas agressivas, quando estas indiquem sofrimento ou prejudiquem intensamente a convivência da pessoa com TEA em seu meio familiar e escolar $[\ldots]$ '. (7).

O Protocolo tem como objetivo fazer com que as Secretarias de Saúde espalhadas por todo o Brasil sigam as determinações presentes na Linha de Cuidado para a Atenção às Pessoas com Transtornos do Espectro do Autismo e suas Famílias na Rede de Atenção Psicossocial do Sistema Único de
Saúde e nas Diretrizes de Atenção à Reabilitação da Pessoa com Transtornos do Espectro Autista, como cita:

"Parágrafo único. O Protocolo de que trata este artigo, que contém o conceito geral do comportamento agressivo como transtorno do espectro do autismo, critérios de diagnóstico, tratamento e mecanismos de regulação, controle e avaliação, é de caráter nacional e deve ser utilizado pelas Secretarias de Saúde dos Estados, Distrito Federal e Municípios na regulação do acesso assistencial, autorização, registro e ressarcimento dos procedimentos correspondentes". (7)

Ainda em 2019 houve a sanção de uma Lei considerada uma conquista por entidades ligadas à luta do TEA: a Lei $\mathrm{n}^{\circ} 13.861$, que prevê a inclusão das especificidades inerentes ao TEA nos censos demográficos (22).

Até o presente momento, as questões do Censo não consideravam o TEA entre as hipóteses de identificação de deficiências. Sendo assim, este não era um dado que entrava para as estatísticas do País. Portanto, o Brasil não possui dados estatísticos de quantos indivíduos brasileiros possuem TEA, nem em qual região ou estado estão localizados, apenas possui estimativas (23).

Esta é uma Lei que contribui de forma significativa para familiares e indivíduos com TEA, pois torna estas pessoas visíveis aos governos federal, estadual e municipal. Além disso, quando houver a contagem destes indivíduos e familiares, isso facilitará o desenvolvimento de políticas públicas mais efetivas, permitindo a maior inclusão dos indivíduos com TEA na sociedade, além de promover maior visibilidade desta condição.

Ainda em 2020, o governo sancionou a Lei mais recente relacionada ao TEA: a Lei $\mathrm{n}^{\circ}$ 13.977. Nessa Lei prevê-se que os estabelecimentos públicos e privados podem utilizar o símbolo da "fita quebra-cabeça" para identificar que indivíduos com TEA têm preferência no atendimento (10). Nesta mesma Lei está prevista a criação da Carteira de Identificação da Pessoa com Transtorno do Espectro Autista (Ciptea): 
[...] “Art. $3^{\circ}-A$. É criada a Carteira de Identificação da Pessoa com Transtorno do Espectro Autista (Ciptea), com vistas a garantir atenção integral, pronto atendimento e prioridade no atendimento e no acesso aos serviços públicos e privados, em especial nas áreas de saúde, educação e assistência social[...]" (10).

Políticas públicas brasileiras estaduais. Em 2011, anteriormente à sanção da Lei $n^{\circ} 12.764$, no Distrito Federal, foi publicada a Lei $n^{\circ} 4.568$ que "institui a obrigatoriedade de o Poder Executivo proporcionar tratamento especializado, educação e assistência especificas a todos os autistas, independentemente de idade, no âmbito do Distrito Federal". A Lei também prevê que será de responsabilidade do Estado o treinamento de profissionais de saúde que atuam no tratamento dos indivíduos com TEA e especializações para alguns cursos de graduação (24).

Em 2015, a Assembleia Legislativa do Estado de São Paulo sancionou a Lei no 15.668 que "dispõe sobre diretrizes para a política de diagnóstico precoce e tratamento dos sintomas da Sindrome do Autismo no âmbito do sistema de saúde". A Lei prevê acesso à medicação, incentivo à pesquisa, programas de acesso ao diagnóstico precoce e tratamento multidisciplinar. Entretanto, não informa a quantidade de recursos a ser disponibilizada e nem como se dará os demais acessos (25).

Em 2018, novamente, o Distrito Federal e São Paulo propuseram outras leis que abrangem os indivíduos com TEA. Em São Paulo, a Lei no 16.756 determina que os estabelecimentos públicos e privados que disponibilizam atendimento prioritário devem incluir os indivíduos com TEA nessa classificação, por meio da disponibilização da "fita quebra-cabeça" nas placas sinalizadoras (26).

No caso do Distrito Federal, por meio da Lei ${ }^{\circ}$ $6.193 / 2018$, indivíduos com TEA foram considerados pessoas com deficiência e, assim, estariam asseguradas pelo atendimento prioritário. Entretanto, não cita que os estabelecimentos devem sinalizar com algum símbolo (27).

Em 2019, o Estado de São Paulo sancionou outra lei: a Lei no 17.158 que "institui a Política Estadual de Proteção dos Direitos da Pessoa com
Transtorno do Espectro Autista". A Lei prevê alguns dos direitos previstos na Lei $n^{0} 12.764 / 2012$, além de incentivo à pesquisa dentro do estado, treinamento de profissionais e a responsabilidade do poder público quanto à informação sobre o TEA (28).

\section{Políticas públicas internacionais (Reino} Unido, Estados Unidos da América e Canadá. Os Quadros 1 e 2 contêm informações sobre políticas públicas adotadas no Reino Unido e EUA. Como o Reino Unido é formado pela junção de alguns países foram reunidas informações de quatro nações: Inglaterra, Escócia, País de Gales e Irlanda do Norte. O intuito foi reunir informações sobre as políticas adotadas na forma de resposta a perguntas formuladas na primeira coluna.

Em novembro de 2012, o Canadá promulgou o World Autism Awareness Day Act. Nessa lei ficou estabelecido que o dia 2 de abril, deve ser conhecido como World Autism Awareness Day (34).

Por meio da publicação do Excise Tax Act (2014) algumas determinações sobre a isenção do imposto GST/HST (Goods And Services Tax/ Harmonized Sales Tax - imposto sobre mercadorias e serviços) ficaram esclarecidas. Sendo assim, serviços que utilizam as ferramentas de acompanhamento comportamental ABA (Applied Behavioural Analysis) ou o IBI (Intensive Behauvioural Intervention) estão isentos do imposto se (1) são utilizadas para auxiliar um indivíduo com autismo; (2) são direcionadas para o indivíduo com autismo ou para alguém que cuide de alguém com autismo e (3) a - As ferramentas são certificadas por psicólogo, médico, assistente social ou enfermeira; $\mathrm{b}-\mathrm{O}$ design do serviço é fornecido pelo governo federal, provincial ou territorial; $\mathrm{c}-\mathrm{A}$ pessoa que cria o escopo de como serão utilizadas as ferramentas é paga pelo governo federal, provincial ou territorial ou por uma organização que administra um programa governamental destinado a ajudar indivíduos com autismo a planejar o treinamento; $\mathrm{d}$ - A pessoa que adquire o serviço das ferramentas é paga pelo governo federal, provincial ou territorial ou por uma organização que administra um programa governamental destinado a ajudar indivíduos com autismo a planejar o uso do $\mathrm{ABA}$ ou do IBI (35). 
Em 2018 foi anunciada a criação do Autism Spectrum Disorder Strategic Fund, que contaria com 91 milhões de dólares por cinco anos (36).

"O fundo foi projetado para apoiar projetos inovadores baseados na comunidade que fornecerão oportunidades tangiveis para os canadenses que vivem com autismo, suas familias e cuidadores adquirirem conhecimentos, recursos $e$ habilidades que possam ajudá-los a enfrentar os desafios de viver com o autismo. Espera-se que, a longo prazo, esses investimentos levem aos melhores resultados de saúde possiveis para pessoas com autismo" (36).

Ainda em 2018 foi publicado o primeiro relatório com dados nacionais e informações para aumentar o conhecimento dos canadenses sobre o TEA. O documento National Autism Spectrum Disorder Surveillance System (NASS) 2018 Report foi publicado com a ajuda do National Autism Spectrum Disorder Surveillance System (NASS). Essa é uma ferramenta, em colaboração dos governos federal, provincial e territorial criada para esclarecer o cenário atual do TEA no Canadá. O NASS possui quatro objetivos: (1) estimar quantas crianças e jovens no Canadá possuem TEA e quantos novos casos estão surgindo ao longo dos anos; (2) descrever a população de crianças e jovens com TEA e comparar padrões canadenses e internacionais; (3) aumentar a conscientização e conhecimento e (4) informar as decisões sobre pesquisas, políticas e programas (37).

No mesmo ano, o orçamento proposto acabou por incluir algumas despesas, ocorridas depois de 2017, relacionadas a animais especialmente treinados para realizar tarefas específicas para algumas deficiências: cegueira, surdez profunda, autismo severo, diabetes severo, epilepsia severa ou comprometimento grave e prolongado que restringe o uso dos braços ou pernas do paciente $(38,39)$.

Em 2020, o governo canadense publicou um relatório que resume iniciativas encontradas através do programa Evaluation of the Opportunities Fund for Persons with Disabilities - Phase I. O programa foi lançado em 1997 com a intenção de cobrir lacunas que o governo federal tenha deixado no suporte a pessoas com deficiências e que não são elegíveis para o programa de seguro desemprego (Employment Insurance). No relatório são citadas duas iniciativas: Ready, Willing and Able e CommunityWorks/Worktopia (40).

Ready, Willing and Able (RWA) é uma parceira nacional entre a Canadian Association for Community Living (CACL), a Canadian Autism Spectrum Disorders Alliance (CASDA) e seus membros. Ela foi projetada para aumentar a mão de obra no mercado de trabalho que possui alguma deficiência intelectual ou TEA. Além de conectar pessoas que estão em busca por um emprego com empregadores, a iniciativa também auxilia empregadores a se tornarem mais inclusivos, coloca em contato empregadores que já contrataram indivíduos com deficiências com aqueles que querem contratar e outros serviços (41).

O Worktopia Project foi fundado em parte pelo governo canadense e sob a liderança dos órgãos The Sinneave Family Foundation e Autism Speaks Canada. Os programas do Worktopia foram projetados para desenvolver habilidades, a fim de melhorar as chances de sucesso no emprego. A iniciativa conta com dois programas: CommunityWorks Canada (CWC) e EmploymentWorks Canada (42).

O primeiro programa tem como foco adolescentes e jovens adultos com autismo, entre 15 e 21 anos, que estão cursando o ensino médio. Após o horário escolar, os participantes têm a oportunidade de desenvolver e praticar habilidades básicas de trabalho, melhorar habilidades sociais e de comunicação, adquirir experiência voluntária da comunidade e cultivar áreas de interesse em potenciais futuros empregos. O segundo programa é um projeto contínuo de treinamento para adultos com autismo e outras deficiências, que os prepara para o mercado de trabalho (42). Em março desse mesmo ano foi divulgado o documento 2020-21 Departmental Plan: Public Health Agency of Canada (PHAC). Nele, a PHAC se comprometeu a investir em projetos inovadores para dar suporte aos canadenses afetados pelo TEA, com foco em iniciativas que possam ajudar esses indivíduos e suas famílias a viverem com bem-estar (43).

A agência também se comprometeu a continuar com o desenvolvimento do Autism-Intellectual 
Disability National Resource and Exchange (AIDE) Network. Esse seria um site nacional e um centro de recursos online para os canadenses. Era esperado que o AIDE Network fosse lançado em março de 2020, mas seu lançamento completo será apenas em setembro de 2021 (44) e esse forneceria acesso online às mais recentes pesquisas, informações, recursos e apoios sobre o TEA (43). Para a criação desse site, o governo canadense disponibilizou 10,9 milhões de dólares (45).

Quadro 1. Políticas públicas no Reino Unido. (29)

\begin{tabular}{|c|c|c|c|c|}
\hline Questão/País & Inglaterra & Escócia & País de Gales & Irlanda do Norte \\
\hline $\begin{array}{l}0 \text { país possui uma lei } \\
\text { geral para o TEA? }\end{array}$ & Autism Act, de 2009. & Scottish Strategy for Autism, de 2011. & $\begin{array}{l}\text { Autistic Spectrum Disorder (ASD) } \\
\text { Strategic Action Plan for Wales, de } \\
2008 .\end{array}$ & $\begin{array}{l}\text { The Autism Act (Nothern Ireland) } \\
2011 .\end{array}$ \\
\hline $\begin{array}{l}0 \text { que é estabelecido no } \\
\text { documento? }\end{array}$ & $\begin{array}{l}0 \text { governo é responsável por: produzir } \\
\text { e realizar revisões periódicas nas } \\
\text { estratégias de auxílio aos indivíduos } \\
\text { com TEA e produzir guias para } \\
\text { as autoridades locais sobre como } \\
\text { implementar essas estratégias em } \\
\text { suas regiões. }\end{array}$ & $\begin{array}{l}0 \text { governo é responsável por: } \\
\text { liderença estratégica para melhorar } \\
\text { a vida das pessoas afetadas pelo } \\
\text { autismo, fornecer o melhor serviço } \\
\text { possível, formar redes de trabalho, } \\
\text { garantir que o diagnóstico de adultos } \\
\text { seja bem conduzido e auxiliar autistas } \\
\text { na busca por emprego. Autistas, } \\
\text { suas famílias e cuidadores devem ser } \\
\text { envolvidos em todos os níveis de } \\
\text { decisões. }\end{array}$ & $\begin{array}{l}\text { Mapear a prevalência, } \\
\text { necessidades e serviços, serviços } \\
\text { de comissionamento, disposições } \\
\text { transitórias (Transitional } \\
\text { arrangements), serviços para adultos } \\
\text { e aumento da conscientização, } \\
\text { informação e treinamento. }\end{array}$ & $\begin{array}{l}0 \text { poder Executivo deve publicar } \\
\text { estratégias para o cuidado de } \\
\text { pacientes com TEA, para todas as } \\
\text { idades, e que seja realizado, a cada } \\
\text { três anos, um relatório dos resultados } \\
\text { das estratégias. }\end{array}$ \\
\hline $\begin{array}{l}\text { Após esse primeiro } \\
\text { documento houve a } \\
\text { publicação de outros, } \\
\text { sem se tratar de uma } \\
\text { atualização? }\end{array}$ & $\begin{array}{l}\text { Primeiro documento com estratégias } \\
\text { de cuidado (2010), com os seguintes } \\
\text { objetivos: aumentar a conscientização } \\
\text { e compreensão do autismo entre } \\
\text { profissionais da saúde, desenvolver } \\
\text { caminhos para o diagnóstico preciso, } \\
\text { aumentar o acesso de adultos autistas } \\
\text { aos serviços e ajudar na busca de } \\
\text { emprego. }\end{array}$ & $\begin{array}{l}\text { Não é citado no documento de } \\
\text { referência. }\end{array}$ & $\begin{array}{l}\text { Não é citado no documento de } \\
\text { referência. }\end{array}$ & $\begin{array}{l}\text { The Autism Strategy (2013-2020) e } \\
\text { o Action Plan (2013-2016), ambos } \\
\text { de 2014. Algumas prioridades } \\
\text { foram definidas no Action Plan: } \\
\text { conscientização, acessibilidade, } \\
\text { saúde e bem-estar, educação, } \\
\text { empregabilidade, independência, } \\
\text { escolhas e controles, acesso à justiça, } \\
\text { ser parte da comunidade, participação } \\
\text { e cidadania ativa. }\end{array}$ \\
\hline $\begin{array}{l}0 \text { país possui uma } \\
\text { diretriz ou guia para os } \\
\text { profissionais de saúde? }\end{array}$ & $\begin{array}{l}\text { Publicado em 2010; pontos } \\
\text { de destaque: treinamento aos } \\
\text { profissionais que lidam com autistas, } \\
\text { diagnóstico de adultos autistas e } \\
\text { planejamento nos serviços prestados. } \\
\text { Atualização em 2015, com novos } \\
\text { objetivos: apoio preventivo de } \\
\text { acordo com o Care Act 2014, apoiar } \\
\text { pessoas com necessidades complexas, } \\
\text { emprego para adultos autistas e } \\
\text { trabalhar em conjunto com o sistema } \\
\text { de justiça criminal. }\end{array}$ & $\begin{array}{l}\text { Não é citado no documento de } \\
\text { referência. }\end{array}$ & $\begin{array}{l}\text { Não é citado no documento de } \\
\text { referência. }\end{array}$ & $\begin{array}{l}\text { Não é citado no documento de } \\
\text { referência. }\end{array}$ \\
\hline $\begin{array}{l}\text { Houve algum tipo de } \\
\text { atualização do primeiro } \\
\text { documento publicado? }\end{array}$ & $\begin{array}{l}\text { Em } 2014 \text { foi publicado o Think Autism } \\
\text { - fulfilling and rewarding lives, the } \\
\text { strategy for adults with autism in } \\
\text { England: an update, com as seguintes } \\
\text { prioridades: construir comunidades } \\
\text { mais conscientes e acessíveis aos } \\
\text { autistas, promover ideias, serviços e } \\
\text { projetos inovadores locais e realçar } \\
\text { como conselhos e informações } \\
\text { sobre serviços podem ser melhores } \\
\text { associados às pessoas. }\end{array}$ & $\begin{array}{l}\text { Scottish Strategy for Autism: outcomes } \\
\text { and priorities 2018-2021, de } 2018 \text {. } \\
\text { Esse documento foi escrito com } \\
\text { base em uma consulta pública para } \\
\text { atualizar as estratégias adotadas } \\
\text { em } 2011 .\end{array}$ & $\begin{array}{l}\text { One-year plan for children, young } \\
\text { people and adults with autism } \\
\text { spectrum disorder, de 2015. Foram } \\
\text { definidas novas prioridades: verificar } \\
\text { atrasos nos diagnósticos de TEA, } \\
\text { desenvolver estratégias para um } \\
\text { serviço integrado, identificar } \\
\text { as necessidades não atendidas, } \\
\text { melhorar os resultados da educação } \\
\text { e do emprego e desenvolver } \\
\text { conscientização e treinamento } \\
\text { profissional. } \\
\text { Em } 2016 \text { foi publicado o documento } \\
\text { Strategic Action Plan for Autism } \\
\text { Spectrum Disorder, cujas prioridades } \\
\text { são: aumento da conscientização, } \\
\text { informação e treinamento, } \\
\text { avaliação e diagnóstico e atender às } \\
\text { necessidades. }\end{array}$ & $\begin{array}{l}\text { Não é citado no documento de } \\
\text { referência. }\end{array}$ \\
\hline Outras ações/publicações & $\begin{array}{l}\text { Em } 2019 \text { foi realizada uma consulta } \\
\text { pública para a revisão do Think } \\
\text { Autism. } \\
0 \text { governo criou o Autism Innovation } \\
\text { Fund, para desenvolver soluções } \\
\text { criativas e econômicas. A verba } \\
\text { arrecada pode ser sorteada e financiar } \\
\text { projetos de inovação em autismo. }\end{array}$ & $\begin{array}{l}\text { Não é citado no documento de } \\
\text { referência. }\end{array}$ & $\begin{array}{l}\text { Em } 2018 \text { foi apresentado o projeto } \\
\text { de lei Autism (Wales) Bill, que não } \\
\text { seguiu em frente. }\end{array}$ & $\begin{array}{l}\text { Em 2016, The National Autistic } \\
\text { Society Nothern Ireland e Autism } \\
\text { NI publicaram o documento Broken } \\
\text { Promises, no qual argumentam que } \\
\text { irlandeses falharam em cumprir as } \\
\text { promessas propostas para indivíduos } \\
\text { com TEA, suas famílias e cuidadores. }\end{array}$ \\
\hline
\end{tabular}


Quadro 2. Políticas públicas nos EUA. (15, 30-33)

\begin{tabular}{|c|c|c|c|c|c|}
\hline Categoria/Ano & 2000 & 2006 & 2011 & 2014 & 2019 \\
\hline $\begin{array}{l}\text { Documento } \\
\text { publicado }\end{array}$ & Children's Health Act (30) & Combating Autism Act (31) & $\begin{array}{l}\text { Combating Autism } \\
\text { Reauthorization Act of } \\
2011 \text { (32) }\end{array}$ & $\begin{array}{l}\text { Autism Collaboration, } \\
\text { Accountability, Research, } \\
\text { Education and Support } \\
\text { (CARES) Act of } 2014 \text { (33) }\end{array}$ & $\begin{array}{l}\text { Autism Collaboration, } \\
\text { Accountability, Research, } \\
\text { Education and Support } \\
\text { (CARES) Act of } 2019 \text { (15) }\end{array}$ \\
\hline Criação de comitês & $\begin{array}{l}\text { Criação do Autism Coordinating } \\
\text { Committee, para coordenar, em } \\
\text { conjunto com o Departamento } \\
\text { de Saúde e Serviços Humanos, } \\
\text { todos os esforços coletados sobre } \\
\text { autismo, incluindo atividades } \\
\text { realizadas pelo National Institutes } \\
\text { of Health (NIH) e Centers for } \\
\text { Disease Control and Prevention } \\
\text { (CDC). Caso o Secretário } \\
\text { acredite ser apropriado, pais } \\
\text { ou responsáveis legais de } \\
\text { indivíduos com TEA podem } \\
\text { participar do comitê. Também } \\
\text { ficou estabelecido que, no dia } \\
1 \text { de janeiro de } 2001 \text { e, a cada } \\
\text { primeiro de janeiro subsequente } \\
\text { a esse ano, deve ser entregue um } \\
\text { relatório sobre a implementação } \\
\text { das medidas e as emendas } \\
\text { realizadas. }\end{array}$ & $\begin{array}{l}\text { Desenvolvimento do Interagency } \\
\text { Autism Coordinating Committee } \\
\text { (IACC), cujas funções são: } \\
\text { coordenar todos os esforços em } \\
\text { conjunto com o Departamento } \\
\text { de Saúde e Serviços Humanos } \\
\text { em relação ao TEA, aumentar } \\
\text { e atualizar os avanços nas } \\
\text { pesquisas, monitorar e fazer } \\
\text { recomendações ao secretário } \\
\text { sobre possíveis intervenções } \\
\text { federais, fazer recomendações } \\
\text { ao secretário sobre participação } \\
\text { pública nas decisões relacionadas } \\
\text { aos transtornos, desenvolver e } \\
\text { atualizar anualmente um plano } \\
\text { estratégico para pesquisa e } \\
\text { submeter ao Congresso esse } \\
\text { plano estratégico e atualizações } \\
\text { relacionadas. }\end{array}$ & - & - & - \\
\hline $\begin{array}{l}\text { Principais medidas } \\
\text { adotadas }\end{array}$ & $\begin{array}{l}\text { Expansão, intensificação e } \\
\text { coordenação das atividades } \\
\text { do NIH nas pesquisas sobre } \\
\text { TEA, programas de pesquisa e } \\
\text { vigilância sobre deficiências no } \\
\text { desenvolvimento, informação e } \\
\text { educação, comitê de coordenação } \\
\text { entre agências de autismo e } \\
\text { relatórios para o Congresso. }\end{array}$ & $\begin{array}{l}0 \text { Departamento de Saúde e } \\
\text { Serviços Humanos, tralhando } \\
\text { em conjunto com o CDC, deve } \\
\text { coletar, analisar e reportar dados } \\
\text { epidemiológicos sobre o TEA e } \\
\text { deficiências do desenvolvimento. } \\
\text { Devem ser criados centros } \\
\text { regionais de excelência para } \\
\text { coletar e analisar informações } \\
\text { sobre números, incidência, } \\
\text { correlatos e causas do TEA e } \\
\text { outras dificuldades relacionadas } \\
\text { ao desenvolvimento. }\end{array}$ & $\begin{array}{l}\text { Permitiu que os trabalhos } \\
\text { do IACC continuassem a } \\
\text { serem realizados. }\end{array}$ & $\begin{array}{l}\text { Reforça as medidas tomadas } \\
\text { anteriormente, com } \\
\text { pequenas alterações. }\end{array}$ & $\begin{array}{l}\text { Nova autorização para o } \\
\text { funcionamento do IACC (até } \\
30 / 09 / 2024 \text { ), orçamento } \\
\text { anual previsto de } 369,7 \\
\text { milhões de dólares norte- } \\
\text { americanos, aumentar o foco } \\
\text { nas atividades do governo } \\
\text { para incluir a vida toda } \\
\text { (lifespan) de uma pessoa com } \\
\text { TEA e exige relatórios sobre } \\
\text { a saúde e o bem-estar dos } \\
\text { mesmos. }\end{array}$ \\
\hline Demais medidas & $\begin{array}{l}0 \text { Secretário de Saúde e Serviços } \\
\text { Humanos (Health and Human } \\
\text { Services) deve estabelecer e } \\
\text { implementar programas que } \\
\text { forneçam educação e informação } \\
\text { sobre o autismo para profissionais } \\
\text { de saúde e para o público em } \\
\text { geral, incluindo o avanço nos } \\
\text { diagnósticos e tratamentos. Além } \\
\text { disso, deve fornecer treinamento e } \\
\text { educação contínua para cientistas, } \\
\text { médicos e outros profissionais que } \\
\text { forneçam cuidados aos autistas. }\end{array}$ & $\begin{array}{l}0 \text { Secretário de Saúde e } \\
\text { Serviços Humanos possui uma } \\
\text { série de responsabilidades: } \\
\text { promover pesquisas para o } \\
\text { desenvolvimento e validação } \\
\text { de ferramentas confiáveis de } \\
\text { triagem, promover a triagem } \\
\text { precoce de indivíduos com maior } \\
\text { risco, aumentar o número de } \\
\text { indivíduos capazes de confirmar } \\
\text { ou descartar um diagnóstico e } \\
\text { fornecer intervenções baseadas } \\
\text { em evidências para pessoas } \\
\text { diagnosticadas, desenvolver } \\
\text { um currículo para a educação } \\
\text { continuada a fim de ajudar } \\
\text { as pessoas a reconhecer } \\
\text { a necessidade e o uso de } \\
\text { ferramentas de triagem válidas } \\
\text { e confiáveis, coletar, armazenar, } \\
\text { coordenar e disponibilizar } \\
\text { publicamente as ferramentas } \\
\text { e outros produtos utilizados } \\
\text { pelos programas federais, } \\
\text { expandir as oportunidades de } \\
\text { treinamento interdisciplinar } \\
\text { existentes ou aumentar o } \\
\text { número de locais capazes de } \\
\text { diagnosticar indivíduos com } \\
\text { TEA ou outras deficiências do } \\
\text { desenvolvimento, promover } \\
\text { a pesquisa de ferramentas } \\
\text { adicionais para reduzir o tempo } \\
\text { de confirmação do diagnóstico } \\
\text { e intervenção precocemente, } \\
\text { desenvolver diretrizes para } \\
\text { essas intervenções e disseminar } \\
\text { informações relacionadas às } \\
\text { pesquisas e diretrizes. }\end{array}$ & - & - & - \\
\hline
\end{tabular}




\section{Outras políticas públicas internacionais. $\mathrm{O}$} Quadro 3 reúne informações sobre políticas públicas internacionais de outros países. As informações de vários países foram condensadas em apenas um único quadro devido as mesmas não serem exten- sas como no Reino Unido, EUA e Canadá. Além disso, algumas informações foram retiradas de artigos científicos, uma vez que os sites governamentais de alguns países não utilizam as línguas inglesa ou portuguesa.

Quadro 3. Políticas públicas na Europa e Austrália. (46-52)

\begin{tabular}{|c|c|c|c|}
\hline País/Questão & Possui uma lei geral para o TEA? & Pontos principais da lei & Outras informações \\
\hline Alemanha & Não. & - & $\begin{array}{l}\text { País segue uma lei geral para } \\
\text { pessoas com deficiência (46). }\end{array}$ \\
\hline França & Não. & - & $\begin{array}{l}\text { País segue uma lei geral para } \\
\text { pessoas com deficiência (46). }\end{array}$ \\
\hline Holanda & Não. & - & $\begin{array}{l}\text { País segue uma lei geral para } \\
\text { pessoas com deficiência (46). }\end{array}$ \\
\hline Itália & $\operatorname{Sim}(47)$ & $\begin{array}{l}0 \text { Ministério da Saúde irá auxiliar e promover pesquisas relacionadas } \\
\text { ao autismo, a fim de compreender sua origem e buscar por } \\
\text { tratamentos. A cada três anos, o Instituto Nacional de Saúde fará } \\
\text { revisão das guidelines do tratamento e inclusão do autismo no LEA } \\
\text { (Livelli Essenziali di Assistenza, serviço nacional de saúde) (48). }\end{array}$ & - \\
\hline Espanha & $\begin{array}{l}\text { Não, mas tem uma diretriz nacional de } \\
\text { cuidados aos autistas ( } 49) \text {. }\end{array}$ & $\begin{array}{l}\text { Aumentar a inclusão social, independência, acessibilidade e } \\
\text { consciência sobre o autismo, fornecer treinamentos aos profissionais } \\
\text { de saúde, investir em pesquisas, garantir o acesso à saúde, educação } \\
\text { e emprego de forma adequada ( } 50) \text {. }\end{array}$ & - \\
\hline Austrália & $\begin{array}{l}\text { Não se trata de uma lei, mas } \\
\text { programas com suporte financeiro } \\
\text { do governo, sendo eles: } \\
\text { o National Disability Agreement (NDA) } \\
\text { e Helping Children with } \\
\text { Autism program (HCWA) (51). }\end{array}$ & $\begin{array}{l}\text { Em 2007, o HCWA foi anunciado com um suporte financeiro de } \$ 190 \\
\text { milhões por cinco anos. } 0 \text { programa tinha como objetivo atingir os } \\
\text { autistas com menos de sete anos e fornecer auxílio financeiro à suas } \\
\text { famílias para o tratamento das crianças. } \\
\text { O NDA também tinha como função fornecer suporte a terapia, } \\
\text { intervenções na infância e/ou intervenção de um especialista, além } \\
\text { de oferecer assistência na busca por emprego (51). }\end{array}$ & $\begin{array}{l}\text { Os dois programas } \\
\text { mencionados devem passar } \\
\text { por um período de transição } \\
\text { até se tornarem o National } \\
\text { Disability Insurance Scheme } \\
\text { (NDIS) (52). }\end{array}$ \\
\hline
\end{tabular}

Ao longo dos anos, tanto o Brasil como outros países vêm tentando aumentar o número de políticas públicas que incluam os indivíduos com TEA. Esse aumento também está relacionado à evolução de pesquisas e descobertas de novas terapias comportamentais, medicamentos que possam diminuir alguns sintomas relacionados aos indivíduos com TEA e novas formas de incluir essas pessoas na sociedade.

Entretanto, as políticas públicas foram apresentadas à sociedade tardiamente, em sua totalidade, após os anos 2000, tanto no Brasil como no exterior, atravancando o desenvolvimento dessas crianças e adultos. Outro ponto que pode ser levantado, as políticas públicas citam crianças, adolescentes e jovens adultos, mas não abordam quais assistências um idoso com TEA precisaria.

Pode-se alegar que as descobertas sobre o transtorno são bastante recentes e que um governo não pode prever quais assistências um idoso com
TEA precisaria. Entretanto, essas pessoas já existem na nossa sociedade e nenhuma política pública enxerga os mesmos.

As leis brasileiras preveem que deve haver incentivo público às pesquisas científicas relacionadas ao TEA, porém essa é uma realidade que pode estar cada vez mais distante da sociedade, uma vez que, os recursos destinados às pesquisas veem sofrendo redução. O mesmo ocorre com o SUS, que seria a maior porta de entrada de famílias de baixa renda com indivíduos com TEA.

Inglaterra, EUA e Canadá têm programas públicos bastante extensos em relação ao TEA. Entretanto, os EUA não possuem um programa público de saúde, como o Brasil (SUS) e a Inglaterra (NHS).

Ainda, destaca-se, de forma positiva, a Estratégia Nacional adotada na Espanha, uma lei específica na Itália e a disponibilização de verbas para suporte aos autistas e seus familiares na Austrália. 
Destaca-se, de forma negativa, a dificuldade e/ou ausência de informação sobre TEA em países como Japão, Alemanha e países nórdicos.

Visto todo o contexto, a atuação do farmacêutico em relação ao TEA é fundamental. Apesar de não ser o profissional que diretamente irá lidar com o paciente autista, o farmacêutico pode ser o primeiro profissional da saúde que entra em contato com a família ou o próprio paciente autista, nas farmácias. Dessa forma, é dever do farmacêutico saber orientar o paciente e a família, além de estar preparado para identificar os primeiros sinais do TEA.

Ainda, por ser o único profissional qualificado para lidar com medicamentos, apenas o farmacêutico, dentro de um grupo multiprofissional, pode elaborar um plano terapêutico para o paciente autista, visando a diminuição de sintomas como ansiedade, agressividade, aumentar a interação social e a fala, bem como avaliar suas pré-condições, avaliando o fármaco com menor impacto de efeitos adversos.

Reforça-se que, além das políticas públicas atuais brasileiras e estrangeiras, abordadas neste trabalho, estarem longe de ser ideais e de promover a inclusão dos indivíduos com TEA na sociedade, o conhecimento sobre este transtorno não está bem elucidado para a população em geral e também para profissionais da saúde.

\section{CONCLUSÃO}

O Transtorno do Espectro Autista é uma condição que acomete milhares de indivíduos e suas famílias. Faz-se necessário a inclusão do TEA entre os transtornos que podem acometer um indivíduo dentro do sistema de saúde do país, assim como as medidas que devem amparar estas pessoas. As políticas públicas estão em constante avanço, porém ainda há muito que evoluir. Também é necessário que o conhecimento sobre o TEA seja igualmente distribuído pela sociedade, mas com foco nos profissionais de saúde, grupo no qual o farmacêutico está incluído. Ele é o único profissional capaz de elaborar um plano terapêutico para o autista, bem como orientar a família como deve ser realizada a medicação e o cuidado com o medicamento, além de ser o primeiro profissional que pacientes possam entrar em contato para buscar auxílio. Desse modo, o farmacêutico pode ser um disseminador de conhecimento sobre o TEA, tornando-se essencial no grupo multiprofissional de tratamento aos autistas.

\section{REFERÊNCIAS}

1. OMS. Autism spectrum disorders. Organização Mundial da Saúde. 2020. Disponível em: https://www.who. int/news-room/fact-sheets/detail/autism-spectrumdisorders

2. AMA-SP. História. 2020. Associação dos Amigos Autistas de São Paulo. Disponível em: https://www.ama. org.br/site/ama/historia/

3. BRASIL. Saúde Mental no SUS: Os Centros de Atenção Psicossocial. Brasília: Ministério da Saúde, Ministério da Saúde. Secretaria de Atenção à Saúde. Departamento de Ações Programáticas Estratégias. 2004. Disponível em: http://www.ccs.saude.gov.br/saude_mental/pdf/ sm_sus.pdf

4. BRASIL. Lei $n^{\circ} 8.069$, de 13 de julho de 1990. Dispõe sobre o Estatuto da Criança e do Adolescente e dá outras providências. Diário Oficial da União, nº 135, 16 de julho de 1990. Seção 1. p. 80-1. Disponível em: http://www. planalto.gov.br/ccivil_03/leis/18069.ht
5. BRASIL. Lei $n^{\circ} 12.764$, de 27 de dezembro de 2012. Institui a Política Nacional de Proteção dos Direitos da Pessoa com Transtorno do Espectro Autista; e altera o $\$ 3^{\circ}$ do art. 98 da Lei $n^{\circ} 8.112$, de 11 de dezembro de 1990 . Diário Oficial da União, n⿳0 250, 28 de dezembro de 2012. Seção 1. p. 1-1. Disponível em: http://www.planalto.gov. br/ccivil_03/_ato2011-2014/2012/lei/112764.htm

6. Oliveira BDCD, Feldman C, Couto MCV, Lima RC. Políticas para o autismo no Brasil: entre a atenção psicossocial e a reabilitação. Physis. 2017;27(3):707-726. DOI: $10.1590 / \mathrm{s} 0103-73312017000300017$.

7. BRASIL. Portaria $n^{\circ} 324$, de 31 de março de 2016. Aprova o Protocolo Clínico e Diretrizes Terapêuticas do Comportamento Agressivo como Transtorno do Espectro do Autismo. Ministério da Saúde. Diário Oficial da União, $n^{\circ} 168,30$ de agosto de 2019. Seção 1. p. 70. Disponível em: https://portalarquivos2.saude.gov.br/images/pdf/2016/ abril/01/Portaria-324-de-31-de-mar--o-de-2016.pdf 
8. BRASIL. Linha de Cuidado para a Atenção às Pessoas com Transtornos do Espectro do Autismo e suas Famílias na Rede de Atenção Psicossocial do Sistema Único de Saúde. Ministério da Saúde. Secretaria de Atenção à Saúde. Departamento de Atenção Especializada e Temática. Brasília: Ministério da Saúde, 2015. Disponível em: http://bvsms.saude.gov.br/bvs/publicacoes/linha cuidado_atencao_pessoas_transtorno.pdf

9. Araujo JAMR, Veras AB, Varella AB. Breves considerações sobre a atenção à pessoa com transtorno do espectro autista na rede pública de saúde. Rev. Psicol. Saúde. 2019;11(1):89-98. DOI: 10.20435/pssa. v11i1.687.

10. BRASIL. Lei $\mathrm{n}^{\circ} 13.977$, de 08 de janeiro de 2020. Altera a Lei $n^{\circ}$ 12.764, de 27 de dezembro de 2012 (Lei Berenice Piana), e a Lei $n^{\circ} 9.265$, de 12 de fevereiro de 1996, para instituir a Carteira de Identificação da Pessoa com Transtorno do Espectro Autista (Ciptea), e dá outras providências. Diário Oficial da União, $n^{\circ} 6,9$ de janeiro de 2020. Seção 1. p. 99-1. Disponível em: http:// www.planalto.gov.br/ccivil_03/_ato2019-2022/2020/lei/ L13977.htm

11. BRASIL. Decreto $n^{\circ} 6.949$, de 25 de agosto de 2009 . Promulga a Convenção Internacional sobre os Direitos das Pessoas com Deficiência e seu Protocolo Facultativo, assinados em Nova York, em 30 de março de 2007. Diário Oficial da União, no 163, 26 de agosto de 2009. Seção 1. p. 104-103. Disponível em: http://www. planalto.gov.br/ccivil_03/_ato2007-2010/2009/decreto/ d6949.htm

12. NAS. Autism Strategy statutory guidance. The National Autistc Society. 2020 Disponível em: https://www. autism.org.uk/about/strategy/statutory-guidance.aspx

13. UNITED KINGDOM. Department of Health. Mental Health, Disability and Dementia Division. Statutory guidance for Local Authorities and NHS organizations to support implementation of the Adult Autism Strategy. London: Department of Health, 2015. Disponível em: https://assets.publishing.service.gov.uk/government/ uploads/system/uploads/attachment_data/file/422338/ autism-guidance.pdf

14. IACC. Autism CARES Act of 2019. Interagency Autism Coordinating Committee. 2020Disponível em: https://iacc.hhs.gov/about-iacc/legislation/autism/caresact-2019/

15. Autism Collaboration, Accountability, Research, Education, and Support Act Of 2019, Pub. L. 116-60, 133 Stat. 1110 (Sept. 30, 2019). Disponível em: https://www. congress.gov/116/plaws/publ60/PLAW-116publ60.pdf

16. Rios C, Ortega F, Zorzanelli R, Nascimento LF. Da invisibilidade à epidemia: a construção narrativa do autismo na mídia impressa brasileira. Interface. 2015; 19(53):325-336. DOI: 10.1590/1807-57622014.0146
17. BRASIL. Portaria n. ${ }^{\circ} 1635 / \mathrm{GM}$, de 12 de setembro de 2002. Inclui, no sistema de informações ambulatoriais do Sistema Único de Saúde - SIA-SUS, os procedimentos relacionados em anexo. Ministério da Saúde. Brasília, DF. Disponível em: http://feapaesp.org.br/material_ download/315_Portaria\%20n.pdf

18. BRASIL. Decreto $\mathrm{n}^{\circ} 8.368$, de 2 de dezembro de 2014. Regulamenta a Lei $n^{\circ} 12.764$, de 27 de dezembro de 2012, que institui a Política Nacional de Proteção dos Direitos da Pessoa com Transtorno do Espectro Autista. Diário Oficial da União, $n^{\circ} 234,3$ de dezembro de 2014. Seção 1. p. 172-2. Disponível em: http:/www.planalto.gov.br/ccivil_03/_Ato2011-2014/ 2014/Decreto/D8368.htm\#: :text=dezembro $\% 20 \mathrm{de} \%$ 201993.-,Art.,infantil\%20at\%C3\%A9\%20a\%20educa\% $\mathrm{C} 3 \% \mathrm{~A} 7 \% \mathrm{C} 3 \% \mathrm{~A} 30 \% 20$ superior

19. BRASIL. Diretrizes de Atenção à Reabilitação da Pessoa com Transtornos do Espectro do Autismo (TEA). Brasília: Ministério da Saúde. Secretaria de Atenção à Saúde. Departamento de Ações Programáticas Estratégicas. 2014. Disponível em: https://bvsms.saude. gov.br/bvs/publicacoes/diretrizes_atencao_reabilitacao_ pessoa_autismo.pdf

20. BRASIL. Lei $\mathrm{n}^{0}$ 10.216, de 6 de abril de 2001 . Dispõe sobre a proteção e os direitos das pessoas portadoras de transtornos mentais e redireciona o modelo assistencial em saúde mental. Diário Oficial da União, no 69, 9 de abril de 2001. Seção 1. p. 1212. Disponível em: http://www.planalto.gov.br/ccivil_03/ LEIS/LEIS_2001/L10216.htm\#: :text=LEI\%20No\%20 $10.216 \% 2 \mathrm{C} \% 20 \mathrm{DE}$,modelo $\% 20$ assistencial $\% 20 \mathrm{em} \% 20$ sa $\%$ C3\%BAde $\% 20$ mental

21. BRASIL. Lei ${ }^{\circ} 10.708$, de 31 de julho de 2003. Institui o auxílio-reabilitação psicossocial para pacientes acometidos de transtornos mentais egressos de internações. Diário Oficial da União, $\mathrm{n}^{\circ}$ 147, 1 de agosto de 2003. Seção 1. p. 72-73. Disponível em: http://www.planalto. gov.br/ccivil 03/Leis/2003/L10.708.htm\#: :text=LEI\% $20 \mathrm{No} \% 2010.708 \% 2 \mathrm{C} \% 20 \mathrm{DE}, \mathrm{Art}$

22. BRASIL. Lei $\mathrm{n}^{\circ} 13.861$, de 18 de julho de 2019. Altera a Lei $n^{\circ} 7.853$, de 24 de outubro de 1989, para incluir as especificidades inerentes ao transtorno do espectro autista nos censos demográficos. Diário Oficial da União, no 138, 19 de julho de 2019. Seção 1. p. 261-1. Disponível em: http://www.planalto.gov.br/ccivil_03/_ ato2019-2022/2019/1ei/L13861.htm

23. Leal S. Nova lei é uma verdadeira vitória para pessoas com autismo. 2020. Disponível em: https://www. selecoes.com.br/colunistas/nova-lei-e-uma-verdadeiravitoria-para-pessoas-com-autismo/

24. DF. Lei $\mathrm{N}^{\mathrm{o}} 4.568$, de 16 de maio de 2011. Institui a obrigatoriedade de o Poder Executivo proporcionar tratamento especializado, educação e assistência específicas a todos os autistas, independentemente de 
idade, no âmbito do Distrito Federal. Distrito Federal. Diário Oficial do Distrito Federal. 26 de maio de 2011. Disponível em: http://www.sinj.df.gov.br/sinj/ Norma/68573/Lei_4568_16_05_2011.html

25. SP. Lei $\mathrm{n}^{\circ} 15.668$, de 12 de janeiro de 2015. Dispõe sobre diretrizes para a política de diagnóstico precoce e tratamento dos sintomas da Síndrome do Autismo no âmbito do sistema de saúde. Diário Oficial do Estado de São Paulo. 13 de janeiro de 2015. São Paulo Disponível em: https://www.al.sp.gov.br/repositorio/legislacao/ lei/2015/lei-15668-12.01.2015.html

26. SP. Lei $\mathrm{n}^{\circ} 16.756$, de 08 de junho de 2018. Dispõe sobre o dever de inserção do símbolo mundial da conscientização sobre o Transtorno do Espectro Autista - TEA nas placas de atendimento prioritário. Diário Oficial do Estado de São Paulo. 9 de junho de 2018. São Paulo Disponível em: https://www.al.sp.gov.br/repositorio/legislacao/lei/2018/ lei-16756-08.06.2018.html

27. DF. Lei $n^{\circ} 6193$, de 16 de outubro de 2018. Altera a Lei $\mathrm{n}^{\circ} 4.027$, de 16 de outubro de 2007, que dispõe sobre a prioridade de atendimento às gestantes, às lactantes, às pessoas acompanhadas de criança no colo, aos idosos com idade igual ou superior a 60 anos, às pessoas com deficiência, às pessoas com obesidade grave ou mórbida, às pessoas que se submetem à hemodiálise e às pessoas portadoras de neoplasia maligna. Diário Oficial do Distrito Federal. 1 de agosto de 2018. Distrito Federal Disponível em: http://www.sinj.df.gov.br/ sinj/Diario/78c3cf68-b4f1-3eb3-a3a8-11839f4e198c/ DODF\%20145\%2001-08-2018\%20INTEGRA.pdf

28. SP. Lei $n^{\circ} 17.158$, de 18 de setembro de 2019. Institui a Política Estadual de Proteção dos Direitos da Pessoa com Transtorno do Espectro Autista - TEA, e dá outras providências. Diário Oficial do Estado de São Paulo. 20 de setembro de 2019. São Paulo Disponível em: https://www.al.sp.gov.br/repositorio/legislacao/lei/2019/ lei-17158-18.09.2019.html

29. Parkin E, Long R, Powell A, Jarrett T. Autism overview of policy and services. House Of Commons Library ["Download full report"; Internet]. 2020 Jan 22. Disponível em: https:/commonslibrary.parliament.uk/ research-briefings/cbp-7172/

30. USA. Children's Health Act Of 2000 , H.R. 4365, $106^{\text {th }}$ Cong. 2000. Disponível em: https://www.govtrack.us/ congress/bills/106/hr4365/text

31. USA. Combating Autism Act of 2006, Pub. L. 109416, 120 Stat. 2821 (Dec. 19, 2006). Disponível em: https://www.congress.gov/109/plaws/publ416/PLAW109publ416.pdf

32. USA. Combating Autism Reauthorization Act of 2011, Pub. L. 112-32, 125 Stat. 361 (Sept. 30, 2011). Disponível em: https:/www.govinfo.gov/content/pkg/ PLAW-112publ32/pdf/PLAW-112publ32.pdf
33. USA. Autism Collaboration, Accountability, Research, Education, and Support Act of 2014, Pub. L. 113-157, 128 Stat. 1831 (Aug. 8, 2014). Disponível em: https:// www.govinfo.gov/content/pkg/PLAW-113publ157/pdf/ PLAW-113publ157.pdf

34. CANADA. World Autism Awareness Day Act Act $\mathrm{n}^{\circ}$ S.C. 2012 , c. 21 , Assented to 2012-11-01. An Act respecting World Autism Awareness Day. Disponível em: https://laws-lois.justice.gc.ca/eng/acts/W-11.6/page1.html\#h-465211

35. CANADA. Canada Revenue Agency. Specially Designed Training to Assist Individuals with Autism. GST/HST Info Sheet GI-113. Disponível em: https://www.canada. ca/en/revenue-agency/services/forms-publications/ publications/gi-113/specially-designed-training-assistindividuals-autism.html

36. CANADA. Public Health Agency of Canada. Funding Opportunities. The Autism Spectrum Disorder Strategic Fund. Disponível em: https://www.canada.ca/en/publichealth/services/funding-opportunities/autism-spectrumdisorder-strategic-fund.html

37. CANADA. Public Health Agency of Canada. Autism Spectrum Disorder among Children and Youth in Canada 2018. A Report of the National Autism Spectrum Disorder Surveillance System. Disponível em: https:// www.canada.ca/en/public-health/services/publications/ diseases-conditions/autism-spectrum-disorder-childrenyouth-canada-2018.html

38. CANADA. Canada Revenue Agency. Details of medical expenses. Disponível em: https:/www.canada.ca/en/ revenue-agency/services/tax/individuals/topics/aboutyour-tax-return/tax-return/completing-a-tax-return/ deductions-credits-expenses/lines-33099-33199eligible-medical-expenses-you-claim-on-your-taxreturn/details-medical-expenses.html\#nmls

39. CANADA. Canada Revenue Agency. Federal government budgets. Budget 2018 - Equality And Growth For A Strong Middle Class. Medical Expense Tax Credit Service Animals. Disponível em: https://www.canada. ca/en/revenue-agency/programs/about-canada-revenueagency-cra/federal-government-budgets/budget-2018equality-growth-strong-middle-class/medical-expensestax-credit.html

40. CANADA. Employment and Social Development Canada. ESDC Evaluation reports. Evaluation of the Opportunities Fund for Persons with Disabilities Phase I. Disponível em: https://www.canada.ca/en/ employment-social-development/corporate/reports/ evaluations/opportunities-fund-persons-disabilities.htm

41. READY, WILLING AND ABLE. Who We Are. Disponível em: http://readywillingable.ca/about/

42. THE WORKTOPIA PROJECT. Our History. Disponível em: https://worktopia.ca/history/ 
43. CANADA. Public Health Agency of Canada. Departmental Plans - Public Health Agency of Canada. 2020-21 Departmental Plan: Public Health Agency of Canada. Disponível em: https://www.canada. ca/en/public-health/corporate/transparency/corporatemanagement-reporting/reports-plans-priorities/20202021-departmental-plan.html

44. AIDE CANADA. How we got started. Disponível em: https://aidecanada.ca/about/history

45. CANADA. Public Health Agency of Canada. Departmental Plans - Public Health Agency of Canada. Public Health Agency of Canada 2020-21 Departmental Plan: Supplementary Information Tables. Disponível em: https:/www.canada.ca/en/public-health/corporate/ transparency/corporate-management-reporting/reportsplans-priorities/2020-2021-supplementary-informationtables.html\#a218

46. Bunt D, Kessel RV, Hoekstra RA, Czabanowska K, Brayne C, Baron-Cohen S, Roman-Urrestarazu A. Quotas, and Anti-discrimination Policies Relating to Autism in the EU: scoping review and policy mapping in Germany, France, Netherlands, United Kingdom, Slovakia, Poland, and Romania. Autism Res. 2020;13(8):1397-1417. DOI: 10.1002/aur.2315

47. ITÁLIA. Legge 18 agosto 2015, n. 134. Disposizioni In Materia di Diagnosi, Cura e Abilitazione Delle Persone Con Disturbi dello Spettro Autistico e di Assistenza
Alle Famiglie. Gazzetta Ufficiale dela Repubblica Italiana, Anno 156 ${ }^{\circ}$, numero 199, 28 ago. 2015. Leggi ed Altri Atti Normativi. p. 5-7. Disponível em: https://www. gazzettaufficiale.it/eli/gu/2015/08/28/199/sg/pdf

48. Gallone D, Siniscalco G. A New Opportunity for Autism: the first specific Italian law. Autism-Open Access. 2015;5(2):135, 2015. DOI: 10.4172/2165-7890. $1000 \mathrm{e} 135$

49. ESPAÑA. Estrategia Española en Trastornos del Espectro del Autismo. Ministerio de Sanidad, Servicios Sociales e Igualdad: Madri, 2015. Disponível em: https:// www.plenainclusion.org/sites/default/files/estrategia espanola_autismo.pdf

50. Autism Europe. 7 set 2020. Spain approves its first National Strategy for Autism. Disponível em: http:// www.autismo.org.es/sites/default/files/tea-01.pdf

51. Roth L. Autism Spectrum Disorder: Briefing Paper No 5/2013. NSW Parliamentary Research Service, 2013. Disponível em: https://www.parliament.nsw. gov.au/researchpapers/Documents/autism-spectrumdisorder/Autism $\% 20$ Spectrum $\% 20$ Disorderbriefing $\% 20$ paper.pdf

52. AUSTRALIA. Autism in Australia. Australian Institute of Health and Welfare. Disponível em: https://www.aihw. gov.au/reports/disability/autism-in-australia/contents/ autism 